\title{
European surveillance for enterovirus D68 during the emerging North-American outbreak in 2014
}

\section{Poelman, Randy}

$2015-10$

Poelman , R, Schuffenecker , I, Van Leer-Buter , C , Josset , L, Niesters, H G M , Lina , B , Vuorinen , T , ESCV-ECDC EV-D68 Study Grp , Lappalainen , M , Jääskeläinen , A \& Smura , T 2015 , ' European surveillance for enterovirus D68 during the emerging North-American outbreak in 2014 ' , Journal of Clinical Virology , vol. 71 , pp. 1-9 . https://doi.org/10.1016/j.jcv.2015.

http://hdl.handle.net/10138/176305

https://doi.org/10.1016/j.jcv.2015.07.296

cc_by_nc_nd

publishedVersion

Downloaded from Helda, University of Helsinki institutional repository.

This is an electronic reprint of the original article.

This reprint may differ from the original in pagination and typographic detail.

Please cite the original version. 


\title{
European surveillance for enterovirus D68 during the emerging North-American outbreak in 2014
}

\author{
Randy Poelman ${ }^{\mathrm{a}, *}$, Isabelle Schuffenecker ${ }^{\mathrm{b}}$, Coretta Van Leer-Buter ${ }^{\mathrm{a}}$, Laurence Josset ${ }^{\mathrm{b}, \mathrm{c}}$, \\ Hubert G.M. Niesters ${ }^{\mathrm{a}}$, Bruno Lina ${ }^{\mathrm{b}, \mathrm{c}}$, on behalf of the ESCV-ECDC EV-D68 study group ${ }^{1}$ \\ a The University of Groningen, University Medical Center Groningen, Department of Medical Microbiology, Division of Clinical Virology, Groningen, \\ The Netherlands \\ ${ }^{\mathrm{b}}$ National Enterovirus Reference Centre, Laboratoire de Virologie, Centre de Biologie Est des Hospices Civils de Lyon, Bron, France \\ c Virpath Lab, EA4610, Faculté de Médecine Lyon Est, Université Claude Bernard Lyon1, Université de Lyon, Lyon, France
}

\section{A R T I C L E I N F O}

\section{Article history:}

Received 7 June 2015

Received in revised form 13 July 2015

Accepted 16 July 2015

\section{Keywords:}

Enterovirus D68

Respiratory infection

VP1 sequencing

Molecular epidemiology

\begin{abstract}
A B S T R A C T
Background: In August and September 2014, unexpected clusters of enterovirus-D68 (EV-D68) infections associated with severe respiratory disease emerged from North-America. In September, the European Centre for Disease Prevention and Control (ECDC) asked European countries to strengthen respiratory sample screening for enterovirus detection and typing in cases with severe respiratory presentations. Objectives: To provide a detailed picture of EV-D68 epidemiology in Europe by conducting a retrospective and prospective laboratory analysis of clinical specimens.

Study design: An initiative supported by the European Society for Clinical Virology (ESCV) and ECDC was launched to screen for EV-D68 in respiratory specimens between July 1st and December 1st 2014 in Europe and to sequence the VP1 region of detected viruses for phylogenetic analytic purposes.

Results: Forty-two institutes, representing 51 laboratories from 17 European countries, analyzed 17,248 specimens yielding 389 EV-D68 positive samples $(2.26 \%)$ in 14 countries. The proportion of positive samples ranged between 0 and $25 \%$ per country. These infections resulted primarily in mild respiratory disease, mainly detected in young children presenting with wheezing and in immuno-compromised adults. The viruses detected in Europe are genetically very similar to those of the North-American epidemic and the majority (83\%) could be assigned to clade B. Except for 3 acute flaccid paralysis (AFP) cases, one death and limited ICU admissions, no severe cases were reported.

Conclusions: The European study showed that EV-D68 circulated in Europe during summer and fall of 2014 with a moderate disease burden and different pathogenic profile compared to the North-American epidemic.
\end{abstract}

(c) 2015 The Authors. Published by Elsevier B.V. This is an open access article under the CC BY-NC-ND license (http://creativecommons.org/licenses/by-nc-nd/4.0/).

\section{Background}

Enterovirus D68 (EV-D68), formerly classified as rhinovirus 87 (RV-87) [14], has a mixture of enterovirus (EV) and rhinovirus (RV) features and can induce severe respiratory symptoms, mostly in children $[15,16]$. In addition, it has been detected in respiratory samples from patients who developed acute flaccid paralysis (AFP)

\footnotetext{
* Corresponding author at: University Medical Center Groningen, Department of Medical Microbiology, Hanzeplein 1, P.O. Box 30 001, 9700 RB Groningen, The Netherlands.

E-mail address: r.poelman@umcg.nl (R. Poelman).

1 The list of participants of ESCV-ECDC EV-D68 study group along with their affiliation are listed in Appendix A.
}

or acute flaccid myelitis [12], but no definite link between EV-D68 infection and neurological disease has yet been established [13].

To date, EV-D68 has never posed a serious threat to the general public health [7-11]. Only limited clusters (in magnitude and in geographic distribution) have been reported in different regions of the world. In most of these instances, the virus was detected in less than $10 \%$ of the EV or RV-related reported cases, and was typically co-circulating with other rhinoviruses or enteroviruses [17-21].

Though, between mid-August and December 2014, an apparent upsurge of severe respiratory tract infections was observed in the United States and Canada, especially among children with underlying chronic pulmonary illness presenting to hospital emergency departments [1-3]. Health authorities in 49 States and the District of Columbia reported 1153 cases of laboratory-confirmed EV-D68 infections by the 15th of January 2015, including 14 fatal cases in young children, and several severe neurological presentations 
(cdc.gov/non-polio-enterovirus/about/ev-d68.html\#outbreak). At the same time, though two European countries reported the circulation of this respiratory virus, no further upsurge of severe respiratory tract infections was reported in Europe ([4-6], ecdc.europa.eu/en/publications/Publications/EnterovirusD68-United-States-Canada.pdf).

Arguably, EV-D68 infections are insufficiently detected owing to a lack of targeted viral diagnostics and clinical awareness [10]. The EV surveillance carried out in Europe, North America and other parts of the world, provides limited information on EV-related respiratory illness. The focus for the worldwide EV surveillance network is primarily on neurological presentation (such as AFP), to support poliovirus eradication programmes. The monitoring of respiratory diseases, carried out by for instance influenza surveillance networks by using pathogen-specific PCRs, is mainly directed towards influenza virus and respiratory syncytial virus during winter. In addition, most hospital-based laboratories are using PCR detection methods which target RV, and may fail to detect EV types [22]. Thus, most of the diagnostic tools used for respiratory virus detection in either in- or out-patients may not detect respiratory EV and, when an RV or an EV is detected by generic PCR assays, no discrimination between these two groups is possible such that the detected viruses are frequently not genotyped. In addition, information about circulating rhinovirus genotypes has been scarcely documented ([23,24], B. Lina personal communication).

\section{Objectives}

In order to provide a detailed picture of the EV-D68 circulation in Europe, we conducted a European-wide retrospective and prospective laboratory analysis of clinical specimens between July and December 2014, in collaboration with the European Society for Clinical Virology (ESCV) and the European Centre for Disease Prevention and Control (ECDC).

\section{Study design}

\subsection{Enrolment of the laboratories}

An ad hoc meeting was held at the annual ESCV conference in Prague (end of September 2014), to launch the European EV-D68 network. Subsequently, the ESCV board invited the members of the scientific community to join this initiative. The ECDC agreed to support in early October and invited all European Union/European Economic Area member states to participate through National Microbiology Focal Points. These efforts resulted in the involvement of a large number of participating laboratories. Also, Quality Control for Molecular Diagnostics was involved as External Quality Assessment Program organization (QCMD EQA, Glasgow, United Kingdom), providing data on performances of commercial assays and laboratory developed tests (LDT's). Forty-two European institutes which represent a total of 51 laboratories from 17 countries (Wales and Scotland representing the United Kingdom) responded to this ESCV/ECDC EV-D68 initiative, of which 35 provided the full set of data (number of specimens received, number of RV-EV positives and number of EV-D68 positives, Table 1). Seven laboratories provided the number of RV-EV positives and number of EV-D68 positives only.

\subsection{Detection of EV-D68 in specimens throughout Europe}

In September 2014, each participating laboratory was asked to test their respiratory specimens collected between the 1st of July and 1 st of December 2014 from children younger than 16 years of age admitted to or visiting the hospital with any respiratory symptoms. Where possible, laboratories were permitted to broaden their retrospective surveillance to before July 1st (i.e., January 2014) and to include samples from adults. Full reporting of the results also had to be completed by December 1st, 2014. For the rapid detection of EV-D68, a specific real-time PCR was developed and proposed by the laboratory in Groningen (NLD) [4]. Each laboratory could use this specific PCR as needed.

\subsection{Sequencing of EV-D68 VP1 in positive specimens and sequence analysis}

Participating laboratories carried out the partial sequencing of VP1 of either EV-D68 positive or EV/RV positive samples as described by Nix et al. [25]. The $\sim 350$ to 400 base pairs VP1 partial sequences obtained were assigned to EV-D68 clades according to Tokarz et al. [26]. Where needed, laboratories could send their material to the Groningen or Lyon laboratories for sequencing.

Sequence data were analyzed with Sequencing Analysis (version 5.3; ABI) using BioNumerics Software 6.6 (Applied Maths, Sint-Martens-Latem, Belgium). Phylogenetic trees (maximum parsimony) were obtained according to BioNumerics standard procedures.

The sequences obtained from the network were aligned using ClustalW in MEGA5 and compared using R bioconductor. Raw distances between pair-wise sequences were calculated using pair-wise deletion method. Amino acids that differentiate each genogroup were defined using custom scripts. Many of the available sequences were deposited in GenBank. The accession numbers are as follows: Denmark (KP729103-KP729109), Finland (KP728259) France (LN626610; LN681316-330; KP196362-378; KP307990; KP406467-496), Germany (KP745729-43), Ireland (KP739245), Italy (KP153538-KP153546), Netherlands (KM887894KM887906/KM924544-KM924547/KM975346-KM975350/ KM975330-KM975335/KP830119-128/KP830131-135), Norway (KP744824-KP744839), Spain (KP090456-KP090459/KP122208), Sweden (KP745744-50).

\subsection{Collection of clinical data}

In addition to the number of specimens processed for this study, each laboratory was asked to provide the number of EV- or $\mathrm{RV}$-detected in-respiratory specimens and the number of EV-D68 positives amongst these specimens.

For the EV-D68 positive cases, the laboratories provided the clinical information blindly, wherever possible, by filling in a short predefined questionnaire. Specifically, participants were requested to report the date of specimen collection, age of the patients, clinical presentation (respiratory illness or other), underlying condition (i.e. chronic respiratory conditions, immuno-compromised status), and admission status (inpatient or outpatient; hospital admission and/or admission to the Intensive Care Unit).

\subsection{Evaluation of the diagnostic tools used in Europe}

Current methods that are used by the participating laboratories for the detection of EV-D68 were assessed. Each laboratory was requested to perform an in silico evaluation of its EV/RV diagnostic procedure for the detection of EV-D68. For this purpose, a sample of the Fermon reference strain was provided by the French National Enterovirus Reference Laboratory to allow laboratories to test the accuracy of their EV or EV/RV assays. Moreover, QCMD-registered laboratories could obtain information on their capacity to detect EV-D68 (this virus being specifically present in the recent EV (2013) and RV (2014) EQA panels.

QCMD has generously provided the performance evaluation sheet of the different techniques used by the participants to the 
Table 1

European EV-D68 detection data by country and laboratory in Europe (July 1st-December 1st 2014), including 916 specimens from a non-screening strategy.

\begin{tabular}{|c|c|c|c|c|c|c|}
\hline Country & Lab and/or city & Tested & $\begin{array}{l}\text { EV Genus (=EV } \\
\text { and/or HRV) }\end{array}$ & EV-D68 & Total/Country & $\begin{array}{l}\text { EV68/EV } \\
\text { Genus (\%) }\end{array}$ \\
\hline \multirow{3}{*}{ Norway } & Oslo NIPH & 66 & 25 & 9 & & \\
\hline & Oslo Ullevål & 354 & 71 & 34 & & \\
\hline & Trondheim & 157 & 101 & 6 & 49 & $24.9 \%$ \\
\hline Sweden & Stockholm Karolinska/PHI/ & $30^{\mathrm{a}}$ & 30 & 7 & 7 & $23.3 \%$ \\
\hline \multirow[t]{3}{*}{ Finland } & Turku UTU & 700 & 140 & 10 & & \\
\hline & Helsinki HUS & 109 & 15 & 4 & & \\
\hline & Helsinki THL & 91 & 29 & 3 & 17 & $9.2 \%$ \\
\hline Scotland & Glasgow & $488^{\mathrm{a}}$ & 488 & 22 & 22 & $4.5 \%$ \\
\hline Ireland & NVRL Dublin & 1010 & 51 & 4 & 4 & $7.8 \%$ \\
\hline Wales & Cardiff NHS & 604 & 62 & 9 & 9 & $14.5 \%$ \\
\hline \multirow[t]{6}{*}{ Netherlands } & Erasmus MC Rotterdam & 198 & 46 & 7 & & \\
\hline & Leiden UMC & 780 & 185 & 3 & & \\
\hline & Radboud UMC & 270 & 6 & 1 & & \\
\hline & Regional health service Haarlem & 252 & 62 & 4 & & \\
\hline & UMC Groningen & 2397 & 531 & 19 & & \\
\hline & RIVM Bilthoven NIH & 370 & 130 & 22 & 56 & $5.8 \%$ \\
\hline Denmark & Statens serum institut SSI & 252 & 50 & 8 & 8 & $16.0 \%$ \\
\hline \multirow[t]{4}{*}{ Germany } & Bonn & 351 & 35 & 1 & & \\
\hline & Freiburg & $136^{\mathrm{a}}$ & 136 & 6 & & \\
\hline & Berlin, Robert Koch Institut ${ }^{\mathrm{C}}$ & 1658 & 58 & 15 & & \\
\hline & Leipzig, Institute of Virology & 364 & 93 & 9 & 31 & $9.6 \%$ \\
\hline Poland & Warsaw NIH & 144 & 22 & 0 & 0 & \\
\hline Romenia & Bucharest & 56 & 0 & 0 & 0 & \\
\hline Austria & Vienna & 778 & 47 & 1 & 1 & $2.1 \%$ \\
\hline Slovenia & Ljubljana & 702 & 234 & 48 & 48 & $20.5 \%$ \\
\hline \multirow[t]{2}{*}{ Italy } & Milan & 176 & 97 & 4 & & \\
\hline & Pavia & $195^{\mathrm{a}}$ & 195 & 9 & 13 & $4.5 \%$ \\
\hline Luxembourg & Luxembourg & $19^{\mathrm{a}}$ & 19 & 1 & 1 & $5.3 \%$ \\
\hline \multirow[t]{9}{*}{ France } & Lyon/Nat ref centre EV & 1589 & 439 & 39 & & \\
\hline & Clermont-Ferrand/Nat ref centre EV & 710 & 191 & 25 & & \\
\hline & Brest & 199 & 86 & 9 & & \\
\hline & Caen & 367 & 175 & 22 & & \\
\hline & Dijon & 159 & 37 & 5 & & \\
\hline & Paris & 49 & 25 & 0 & & \\
\hline & Saint-Etienne & 104 & 36 & 6 & & \\
\hline & Strasbourg & 203 & 102 & 9 & & \\
\hline & Versailles & 109 & 34 & 2 & 117 & $10.4 \%$ \\
\hline \multirow[t]{4}{*}{ Spain } & Barcelona & 529 & 53 & 5 & & \\
\hline & Valencia & 458 & 89 & 0 & & \\
\hline & Santander & $15^{\mathrm{a}}$ & 15 & 0 & & \\
\hline & Valladolid & $33^{a}$ & 33 & 1 & 6 & $3.2 \%$ \\
\hline Portugal & Lisbon & 17 & 0 & 0 & 0 & \\
\hline Total number of countries ${ }^{b}$ & Total number of labs & & & & & \\
\hline 17 & 42 & 17,248 & 4273 & 389 & & \\
\hline
\end{tabular}

a Non-screening strategy.

b Wales and Scotland representing the United Kingdom.

c Includes three laboratories from: Dusseldorf, Heidelberg and Wurzburg.

EV QCMD Quality Control panel. This information was correlated with the in silico self-evaluation provided by the participating labs.

\subsection{Statistical analysis}

Statistical analysis was performed by using the Chi-Square Test (CI 95\%) and Fisher's exact test ( $p$-value $<0.05$ ).

\section{Results}

\subsection{EV-D68 detection and distribution in Europe}

A total of 17,248 (mostly respiratory) samples were tested, mainly collected between the 1 st of July and the 1 st of December
(Table 1). Overall, a picornavirus (EV or RV) was detected in 4273 specimens, of which 389 were identified as EV-D68. The European mean EV/RV positivity rate (calculated from the 35 labs) in respiratory specimens was $20.5 \%$ (3357/16,332), of which $10.21 \%$ (343/3357) were EV-D68 positive, yielding an EV-D68 positivity rate of $2.1 \%(343 / 16,332)$ in respiratory samples.

The virus was detected in each participating country across Europe, except for three: Poland, Portugal and Romania; but in these countries only a limited number of samples were tested. The proportion of EV-D68 positive samples among the total number of respiratory samples tested differed between countries, ranging from $0 \%$ to $25 \%$ (Table 1, Fig. 1). EV-D68 was detected throughout the five months of the study, with a peak in October and a clear 


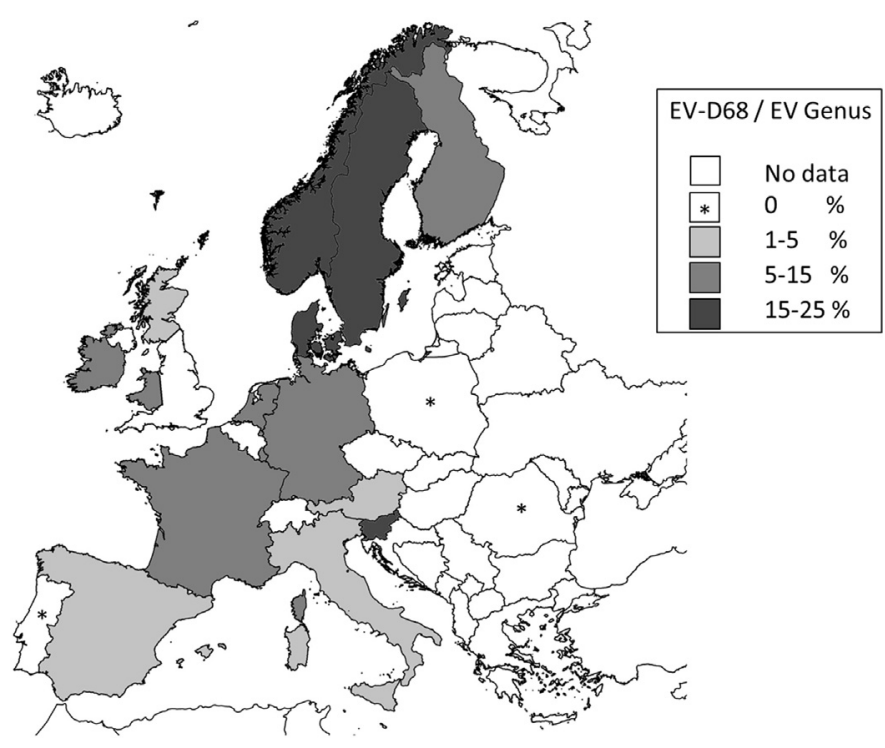

Fig. 1. European detection rates of EV-D68 by participating countries, in Europe, 2014. Colour codes: white non-data, White with a star: no detection, light grey: $1-5 \%$; grey: $5-15 \%$; dark grey $15-25 \%$.

epidemic trend (Fig. 2). A North-South gradient was observed with a higher level of detection in Northern (Norway, Sweden, Denmark) than in Southern European countries (Italy, Spain, Portugal; Fig. 1; Table 1). Only 6 cases were detected in Spain, 13 in Italy and none in Portugal.

\subsection{Sequencing}

Of the 389 EV-D68 strains that were detected, 205 (52.7\%) were sequenced and subsequently assigned to the subgroups described by Tokarz et al. [26]. All the EV-D68 viruses belonged to clades A $(n=35 ; 17.1 \%)$ and B $(n=170 ; 82.9 \%)$; no clade $\mathrm{C}$ virus has been detected. Viruses from the clades A and B were co-detected in 12 countries, while Spain reported only clade A viruses and Slovenia and Norway only clade B viruses. The Blast analysis and the maximum parsimony tree show high nucleotide homology between the US and European viruses (Fig. 3, Suppl. Figure 1). Identical sequences were obtained from viruses detected in different countries (Fig. 3, Suppl. Figure 1). Both figures show a segregation of the clade A viruses into two sub-clusters, A1 ( $n=11$; $31.4 \%)$ and $A 2(n=24 ; 68.6 \%)$ with an average raw nucleotide distance of $11 \%$ between the two sub-clusters (Fig. 3). The significance of this segregation needs to be confirmed by further Bayesian analysis. The nucleotide divergence between the whole clade $A$ and clade B was $13.8 \%$. All 2014 VP1 sequences of clade A viruses had a deletion of one amino acid in position 140, while 9 other aminoacids could differentiate at least one clade from another (Suppl. Table 1). More than 30 amino acids at the end of the VP1 coding sequence were found to be specific to the 2014 sequences when compared to previous sequences (data not shown). These changes are observed in regions where neither the impact on the immunogenicity of the virus nor that on binding to sialic acids was known [27]. Finally, there were no absolute differences in the BC and DE loops of VP1 between the US and the European 2014 viruses (Suppl. Table 2). However, when comparing the frequencies of amino-acids at each position, two positions in the BC and DE loops, at residues 97 and 143 , respectively, showed significant differences between the USA and European strains in Clade B (Fisher's exact test; $p<0.05$ ). As there were only a few viral sequences from the USA outbreak, further analysis must be done before it can be concluded that the strains circulating in the USA and Europe in 2014 were different. Such a comparison could not be performed for viruses of the sub-clade A1 for which no sequence from the USA outbreak was available.

Supplementary Fig. 1, Tables 1 and 2 related to this article can be found, in the online version, at http://dx.doi.org/10.1016/j.jcv. 2015.07.296

\subsection{Clinical analysis}

EV-D68 was detected in all age groups (Table 2). From the 389 documented cases, three AFP cases (two in Norway and one in France) and one death, of a 14-year-old Italian patient with severe neurological underlying disease, have already been reported [40]. Underlying conditions that predisposes to the development of severe respiratory infections were frequent in all age groups (Table 2, Suppl. Table 3). Data on underlying conditions were

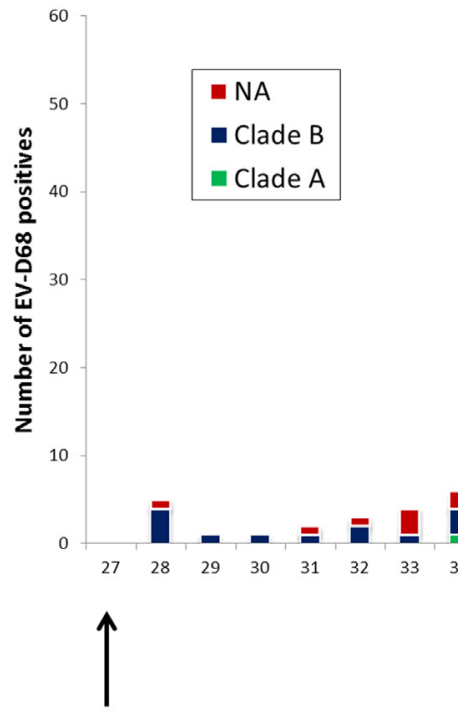

July 1st

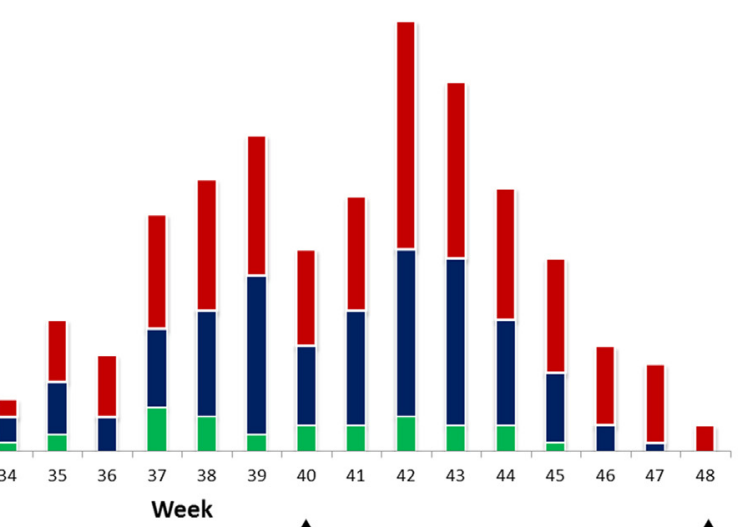

Week

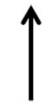

ESCV 2014, Prague

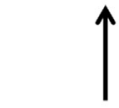

December 1st

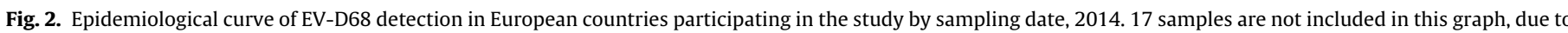
a lack of sampling date. Colour code: Green = clade A: Blue = clade B, Red = clade not assigned. 


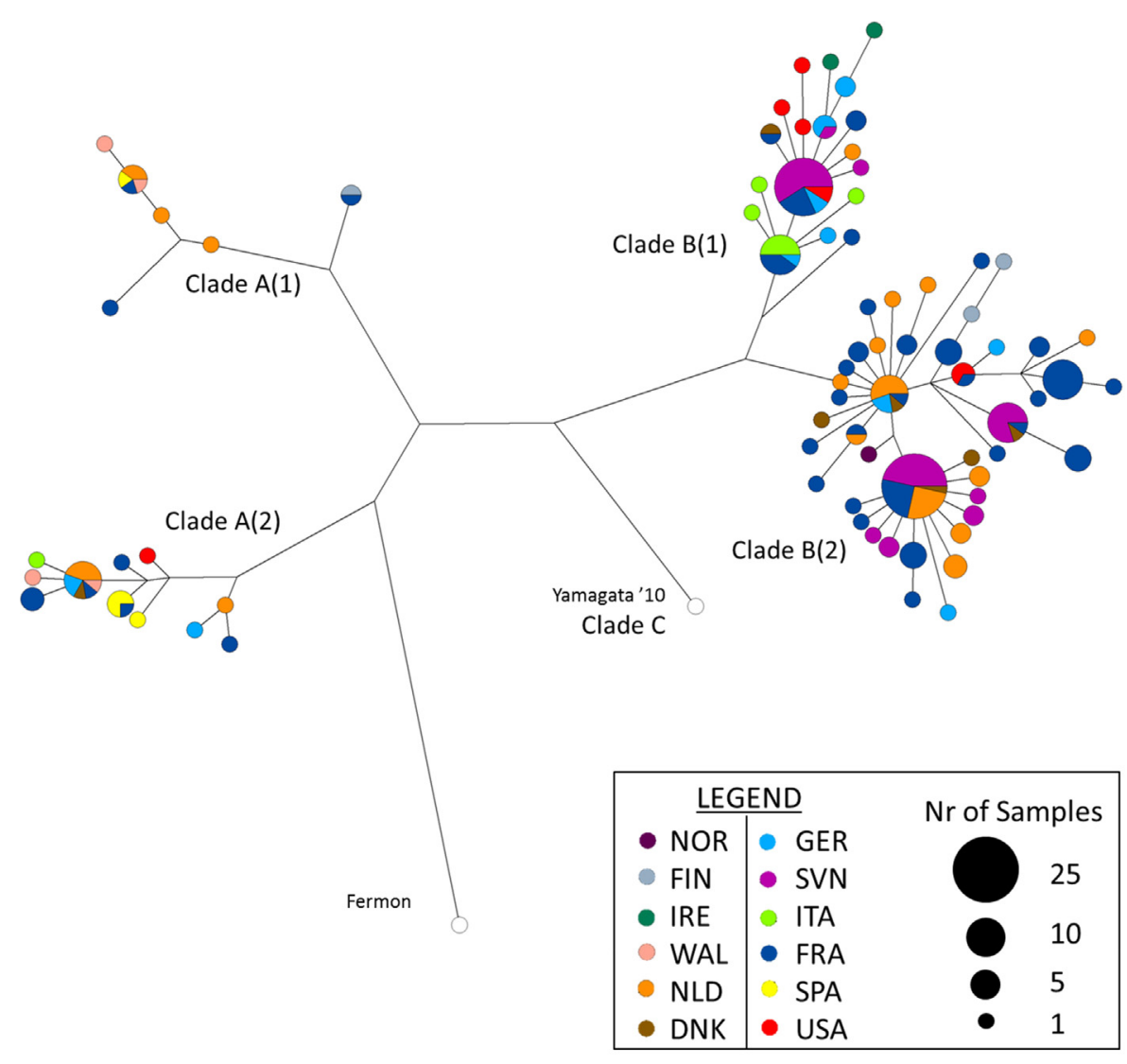

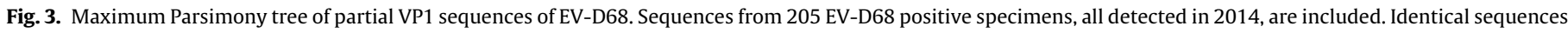
are merged in the bigger nodes. Colour codes for each country are the same as for Fig. 3 and Suppl. Figure 1. Clades are according to Tokarz et al. [26].

available in only $91 / 120$ (76\%) children below the age of 2 . Of these $20 / 91$ (21.9\%) had chronic or recurrent respiratory problems, 10 (11\%) had other underlying disease, 3 (3.3\%) where immuno-compromised, whereas the majority $(58 / 91 ; 63.7 \%)$ did not have any recognized underlying disease (Table 2, Suppl. Table 3 ). In the age group $2-5$ years information was missing from 63 individuals (46.7\%). When documented, $44 / 72$ (61.1\%) were known to have underlying conditions, the largest group having a chronic or recurrent pulmonary disease $(n=27 / 72,37.5 \%)$. In 197 documented children of all age groups (<17), immuno-compromised conditions were present in only 8 of EV-D68 cases (4.1\%). This was different for the adults $(>17)$ in question, where immuno-compromised patients (following transplantation or cancer treatment) formed a significantly larger proportion of the cohort than with the children (37.5\% vs $4.1 \%$; $p<0.001$; Chi-square test). Nonetheless, although the difference is not significant, only $9 / 45$ documented adults (20\%) had underlying chronic respiratory diseases as compared to 63/197 documented children (32\%).

Supplementary Table 3 related to this article can be found, in the online version, at http://dx.doi.org/10.1016/j.jcv.2015.07.296

In the four age groups, similar proportions of patients admitted to the ICU amongst the hospitalized cases were observed (15/110,
$11 / 121,5 / 45$ and 3/38 for 0-1 year, 2-5 year, 6-16 and 17 and over, respectively). None of the clades was associated with clinical severity or age-distribution.

\subsection{Performance of commercial test kits and (specific) real-time $P C R$}

Overall, 29 laboratories used LDT's, 18 used commercial assays and 3 used both in-house and commercial assays. One laboratory did not provide details of the assay used. Briefly, the retrospective analysis of the performance of the commercially available kits confirmed that the SEEGENE Anyplex II RV16 detection assay (Korea) that is used by two laboratories did not detect EV-D68, while this assay preferentially targeting only EV. The Cepheid Smartcycler (Cepheid, Sunnyvale, USA), the Fast-Track Diagnostics (Sliema, Malta) and the Diagenode human EV test (Liege, Belgium), did detect EV-D68. In addition, the most commonly used commercial assays, such as the xTAG Respiratory Viral Panel (Luminex Europe, Den Bosch, The Netherlands) Respifinder Smart 22 fast v2 (Pathofinder, Maastricht, The Netherlands), Rhino\&Entero/Ccr-gene (bioMerieux/Argene, Marcy l'Etoile, France), were not able to discriminate between RV

Table 2

Underlying diseases categories in EV-D68-positive cases by age group in the 14 EU/EEA countries (July 1st-December 1st 2014).

\begin{tabular}{|c|c|c|c|c|c|c|}
\hline \multirow[t]{2}{*}{ Age } & \multirow[t]{2}{*}{ EV68 positive } & \multicolumn{5}{|l|}{ Underlying disease } \\
\hline & & Chronic respiratory illness & Immunocompromised & Non-respiratory illness & None & Not assigned \\
\hline $0-1$ & 120 & $20(16.7 \%)$ & $3(2.5 \%)$ & $10(8.3 \%)$ & $58(48.3 \%)$ & $29(24.2 \%)$ \\
\hline $2-5$ & 135 & $27(20.0 \%)$ & $5(3.7 \%)$ & $12(8.9 \%)$ & $28(20.7 \%)$ & $63(46.7 \%)$ \\
\hline $6-16$ & 56 & $16(28.6 \%)$ & $1(1.8 \%)$ & $6(10.7 \%)$ & $11(19.6 \%)$ & $22(39.3 \%)$ \\
\hline$\geq 17$ & 78 & $9(11.5 \%)$ & $17(21.8 \%)$ & $5(6.4 \%)$ & $14(17.9 \%)$ & $33(42.3 \%)$ \\
\hline Total & 389 & $72(18.5 \%)$ & $26(6.7 \%)$ & $33(8.5 \%)$ & $111(28.5 \%)$ & $147(37.8 \%)$ \\
\hline
\end{tabular}


and EV, but detected EV-68. Specific mentioning that such kits may or may not detect EV-D68 is not included in their product information insert, but testing the Fermon strain did indicate the detection potential of some of these assays. Besides, a total of 31 laboratories implemented one of the available specific EV-D68 real-time PCR's $[4,6]$.

\section{Discussion}

Between 1963 and 2014, EV-D68 upsurges involving children with underlying respiratory diseases have been reported in different countries, albeit with a quite limited number of cases $[8,18,28,29]$. In contrast to the rapidly accumulating EV-D68 cases observed in North America in 2014 [1,11], no sudden increase of EV-D68 was reported in Europe, except for Norway and the Netherlands [4-6]. The initiative launched by the European Society for Clinical Virology (ESCV) and supported by the European Centre for Disease prevention and Control (ECDC) was primarily aimed at a rapid European-wide screening for the detection of the possibly emerging EV-D68.

Following the launch of the initiative, EV-D68 detection protocols were formulated and implemented to screen 17,248 respiratory specimens collected between July and November 2014, allowing a rapid response within a two-month period (October-December 2014). EV-D68 was detected in all participating countries, except for three (Fig. 1, Table 1), though two analyzed a limited number of specimens (17 and 56, respectively) and the other used an assay which may have been the cause for missing (a low level of circulation of) the virus. The 2014 instance was the largest European EV-D68 epidemic described since the isolation of the Fermon strain in 1962.

The trend of detection (though somewhat biased by the implementation of the survey itself) showed a clear epidemic curve, with a peak of cases in October (Fig. 2) and local epidemics reported [4-6]. The number of cases diminished in December, as would be expected for an EV epidemic, particularly where RSV and influenza epidemics come into play [30].

A European North-South gradient was observed, revealing discrepancies in the levels of virus detection. In some instances, discrepancies were observed between laboratories in the same country (Table 1 ). This could be partly explained by local epidemics or clusters (as in Norway and Slovenia) or by local differences in testing periods (the Netherlands). This divergence in observed results raised questions about the capacity of some assays to detect EV-D68. Thanks to the recent EV-RV EQA panels provided by QCMD, which included EV-D68, we could confirm that most of the participating laboratories used kits appropriate for EV-D68 detection. This was further attested when they used the EV-D68-specific detection protocols. However, a few laboratories did not; the Seegene Anyplex II RV16 detection assay ${ }^{\circledR}$ failed to detect EV-D68.

Still, relatively few clinical diagnostic laboratories employ systematic EV detection followed by genotyping in the routine screening strategy of respiratory materials, both in Europe and the USA $[11,16,29]$. Hence, clusters of EV infections may remain undocumented until a significant EV-related clinical burden arises.

The clinical picture that we describe is a reflection of the underlying hospitalized population, rather than a representation of the community or of the primary healthcare level. Consistently about $10 \%$ of the cases in all age groups required intensive care respiratory support. Of the EV-D68 cases recorded in our study, preexisting respiratory conditions were frequent in young children, whereas underlying immune-compromising conditions were frequent in adults (Table 2). This finding has been reported elsewhere [31].
In contrast to the USA, only one death was reported in Europe during this EV-D68 survey and only 3 children were diagnosed with EV-D68 related AFP $[2,6,36]$. This difference in clinical impact was also noted in the recent retrospective study conducted in the USA. Greninger et al. reported that EV-D68 of clade B similar to those detected in the European survey was frequently detected in the respiratory secretions of patients with acute flaccid myelitis [12]. No such cases have been observed in Europe to date. This absence is not attributable either to a lack of detection capabilities or of appropriate sample collection. Indeed, most European countries have active surveillance programmes for poliovirus infections in which EV-associated neurological presentations are reported and documented by reference laboratories [22]. Three cases of EV-D68 related AFP have been reported (two in Norway and one in France), showing the capacity of the system to detect AFP-related cases [36]. Even if no link is established between AFP and EV-D68 infection, more surveillance is needed to better assess its putative role in severe neurological presentations [12,13,37].

As part of our study, 205 isolates were sequenced to investigate their relationship with the circulating EV-D68 virus types in North America and to determine if severity could be correlated to a given clade of the virus [26]. Our sequence data confirms that $A$ and B clades were circulating, just as in the USA and Canada [2]. The European and North American EV-D68 viruses were genetically closely related, but a specific virus genetic signature was not associated with virulence.

EV-D68 can be easily transmitted through droplets and is the most frequently encountered group D EV (although this is a small group) $[11,14,15]$. It has been demonstrated that this virus binds to the sialic acid $\alpha 2,6$, as do other respiratory viruses, as a result of adaptations in the BC and DE loops of the VP1 capsid region [27]. Despite ongoing research, there is no antiviral treatment available [32,33]. However, Lui et al. recently suggested that Pleconaril could be effective in treating the latest EV-D68 infections because of its excellent binding into the hydrophobic canyon of the capsid; this canyon being narrower than those in most other RVs and EVs [34]. Though this drug is not commercially available, it may be useful to evaluate its contribution to the management of severe EV-D68 infections, and the alleviation of outbreaks such as those occurring in nursing homes [24,35].

However, more sequences from the US outbreak are required to determine whether specific residues in the BC and DE loops of VP1 might have different amino acid proportions in US versus European sequences [2]. Despite this apparent lack of difference in the severity of the diseases, some evolution was observed in the BC loop which reinforces the argument in favour of distinguishing between clades A and B $[38,39]$.

In conclusion, this collaborative venture highlights the merits of working together on cross-border studies of infectious disease epidemics using established networks. A timely response is essential to evaluate the circulation and relevance of an emerging virus. Therefore the global or at least continental networks, such as the ECDC and ESCV could provide, need to be equipped to facilitate and streamline proper procedures for a rapid and efficient handling of an emerging virus, whenever it occurs. Additionally, long-term studies are needed to adequately collect clinical data for a better understanding of the pathogenesis.

\section{Conflict of interest}

The authors declare that there is no conflict of interest.

\section{Funding}

None. 


\section{Ethical approval}

None required.

Appendix A. List of participants ESCV-ECDC Enterovirus D68 ad-hoc study group

\section{A.1. Austria}

Theresia Popow-Kraupp, Stephan W. Aberle - (Department of Virology, Medical University of Vienna).

\section{A.2. Denmark:}

Thea Kølsen Fischer - (Virus Surveillance and Research Unit, Department of Microbiological Diagnostics and Virology, Statens Serum Institut and Clinical Institute, University of Southern Denmark).

Sofie Midgley - (National WHO Polio Reference Laboratory at Statens Serum Institut).

Claus Bohn Christiansen - (Virology Unit, Department of Clinical Microbiology at Rigshospitalet).

\section{A.3. Finland}

Matti Waris, Riikka Österback, Tytti Vuorinen - (Department of Virology, University of Turku).

Carita Savolainen-Kopra, Francesca Latronico\#, Soile Blomqvist, Niina Ikonen - (National Institute for Health and Welfare, Virology unit, Helsinki); (\#*European Public Health Microbiology training programme (EUPHEM), European Centre for Disease Prevention and Control, Stockholm, Sweden).

Maija Lappalainen, Anne Jääskeläinen, Teemu Smura (Department of Virology and Immunology, University of Helsinki and Helsinki University Hospital).

\section{A.4. France}

Léa Pilorge, Marie-Christine Legrand-Quillien, Christopher Payan - (Microbiology laboratory, University hospital of Brest).

Joëlle Petitjean, Astrid Vabret, Mélanie Ribault - (Virology laboratory, University hospital of Caen).

Audrey Mirand, Hélène Peigue-Lafeuille, Cécile Henquell (National Reference Center for Enteroviruses and Parechoviruses, University Hospital of Clermont-Ferrand).

Catherine Manoha, Jean-Baptiste Bour, Magali Darniot (Virology laboratory, University hospital of Dijon).

Isabelle Schuffenecker, Laurence Josset, Bruno Lina - (Virology laboratory, National Reference Center for Enteroviruses and Parechoviruses, Hospices Civils de Lyon).

Jérôme Le Goff, Séverine Mercier-Delarue, Catherine Scieux - (Université Paris Diderot, Sorbonne Paris Cité, Microbiology laboratory, Hôpital Saint-Louis, APHP, Paris).

Sylvie Pillet, Bruno Pozzetto - (Laboratory of infectious agents and hygiene, University Hospital of Saint-Etienne, Saint-Etienne).

Quentin Lepiller, Samira Fafi-Kremer, Françoise Stoll-Keller - (Virology institute, University hospital of Strasbourg).

Stéphanie Marque-Juillet; Aymeric Coutard, Marlène Amara - (Microbiology laboratory, Hospital of Versailles).

\section{A.5. Germany}

Sindy Böttcher, Sabine Diedrich - (Berlin, Robert Koch Institute, National Reference Centre for Poliomyelitis and Enteroviruses); The Robert Koch Institute collated data from the following Departments and Institutes: Institute of Virology and
Immunobiology, University Hospital of Würzburg, Christiane Prifert, Benedikt Weißbrich; Institute of Virology, University Hospital of Düsseldorf, Ortwin Adams; Department of Virology, Labor Berlin Charite Vivantes $\mathrm{GmbH}$ ), Angela Stein, Jörg Hofmann; Department of Infectious Diseases, Virology, University Hospital Heidelberg, Paus Schnitzler.

Anna Maria Eis-Hübinger, Souhaib Aldabbagh, Ulrike Reber - (Institute of Virology, University of Bonn Medical Centre).

Marcus Panning, Daniela Huzly, Sibylle Bierbaum - (Institute of Virology, Albert-Ludwigs University Freiburg).

Uwe G. Liebert, Melanie Maier - (Institute of Virology, University of Leipzig).

\section{A.6. Ireland}

Michael J. Carr, Gráinne Tuite, Jorge Abboud Guerra,Joanne O'Gorman, Cillian De Gascun - (National Virus Reference Laboratory, University College Dublin).

\section{A.7. Italy}

Fausto Baldanti - (University of Pavia, Fondazione IRCCS Policlinico San Matteo, Pavia).

Antonio Piralla - (Fondazione IRCCS Policlinico San Matteo, Pavia).

Susanna Esposito, Nicola Principi, Luca Ruggiero - (Milan, Fondazione IRCCS Ca' Granda Ospedale Maggiore Policlinico, Pediatric Highly Intensive Care Unit).

\section{A.8. Luxembourg}

Matthias Opp - (Dudelange, Laboratoire National de Santé).

\section{A.9. Netherlands}

Gé Donker - (Utrecht, Netherlands institute for health services research).

Adam Meijer, Harrie van der Avoort, Kimberley Benschop - (Bilthoven, Centre for Infectious Disease Research, Diagnostics and Screening, National Institute of Public Health and the Environment).

Marit de Lange - (Bilthoven, Centre for Infectious Diseases, Epidemiology and Surveillance, National Institute of Public Health).

Hubert Niesters, Randy Poelman, Coretta Van Leer-Buter, Renze Borger, Liesbeth Scholvinck - (University Medical Center Groningen).

Wil van der Reijden, Dick Veenendaal - (Haarlem, Regional Laboratory for Public Health Kennemerland).

Eric C.J. Claas, Ann C.T.M. Vossen - (Department of Medical Microbiology, Leiden University Medical Center Leiden).

Janette Rahamat-Langendoen, Willem J.G. Melchers (Nijmegen, Department of Medical Microbiology, Radboud University Medical Center).

Marion P.G. Koopmans, Annemiek A. van der Eijk, Suzan D. Pas - (Rotterdam, ErasmusMC, Department of Viroscience).

\section{A.10. Norway}

Anne-Marte Bakken Kran - (Department of Medical Microbiology, Oslo University Hospital).

Karoline Bragstad, Susanne Gjeruldsen Dudman - (Oslo, Norwegian Institute of Public Health).

Andreas Christensen, Sidsel Krokstad - (Trondheim, Department of Medical Microbiology, St. Olavs University Hospital). 


\section{A.11. Poland}

Katarzyna Pancer, Edyta Abramczuk - (National Institute of Public Health-NIH Warsaw).

\section{A.12. Portugal}

Raquel Guiomar, Pedro Pechirra, Paula Cristovão, Ines Costa - (Lisbon, National Influenza Reference Laboratory).

\section{A.13. Romania}

Cristina Tecu, Emilia Lupulescu, Carmen Cherciu - (Bucharest, Cantacuzino National Research and Development Institute for Microbiology and Immunology).

\section{A.14. Scotland}

Emily Goldstein, Susan Bennett, Amanda Bradley-Stewart, Rory Gunson - (West of Scotland Specialist Virology Centre Glasgow).

\section{A.15. Slovenia}

Natasa Berginc, Katarina Prosenc - (Ljubljana, Laboratory for Public Health Virology. National Laboratory of Health, Environment and Food).

\section{A.16. Spain}

Magda Campins, Laura Gimferrer, Andres Anton - (Respiratory Virus Unit/Virology Section, University Hospital Vall d'Hebron, Barcelona).

F. Xavier López-Labrador; Laura Cano Pérez - (Virology Laboratory, Genomics and Health Area, FISABIO-Public Health, Generalitat Valenciana, Valencia/CIBERESP, Instituto de Salud Carlos III).

Joan Puig-Barberá - (Vaccines Research Area, FISABIO-Public Health, Generalitat Valenciana, Valencia).

Concepción Gimeno Cardona, M Dolores Ocete Mochón (Microbiology Service, General Hospital of Valencia).

Javier Buesa, David Navarro - (Microbiology Service, Hospital Clinico of Valencia).

R. Ortiz de Lejarazu Leonardo, Iván Sanz Muñoz, Silvia Rojo (University Hospital of Valladolid).

Mónica Gozalo-Margüello, Jesús Agüero Balbín - (Service of Microbiology, University Hospital Marqués de Valdecilla-IDIVAL, Santander).

\section{A.17. Sweden}

Jan Albert, Agneta Samuelson, Maria Rotzén Östlund, Robert Dyrdak - (Stockholm, Department of Clinical Microbiology, Karolinska University Hospital).

Mia Brytting, Elenor Hauzenberger, Katherina Zakikhany (Stockholm, Public Health Agency of Sweden).

Margareta Eriksson - (Stockholm, Astrid Lindgren Children's Hospital).

\section{A.18. Wales}

Catherine Moore - (Public Health Wales Microbiology Cardiff, University Hospital of Wales).
A.19. ECDC

Eeva Broberg, Pasi Penttinen - European Centre for Disease Prevention and Control (Stockholm Sweden)

A.20. QCMD

Elaine McCulloch, Caterina Di Lorenzo, Paul Wallace, Anton Van Loon - Quality Control for Molecular Diagnostics (Scotland/United Kingdom).

\section{References}

[1] C.M. Midgley, M.A. Jackson, R. Selvarangan, G. Turabelidze, E. Obringer, D. Johnson, B.L. Giles, A. Patel, F. Echols, M.S. Oberste, W.A. Nix, J.T. Watson, S.I Gerber, Severe respiratory illness associated with enterovirus D68 - Missour and Illinois, 2014, MMWR Morb. Mortal. Wkly. Rep. 63 (September (36)) (2014) 798-799.

[2] J. Stephenson, CDC tracking enterovirus D-68 outbreak causing severe respiratory illness in children in the Midwest, JAMA 312 (October (13)) (2014) 1290

[3] B.A. Brown, W.A. Nix, M. Sheth, M. Frace, M.S. Oberste, Seven strains of enterovirus D68 detected in the United States during the 2014 severe respiratory disease outbreak, Genome Announc. 2 (November (6)) (2014), pii: e01201-14.

[4] R. Poelman, E.H. Schölvinck, R. Borger, H.G. Niesters, C. van Leer-Buter, The emergence of enterovirus D68 in a Dutch University Medical Center and the necessity for routinely screening for respiratory viruses, J. Clin. Virol. 62 (January) (2015) 1-5.

[5] A. Meijer, K.S. Benschop, G.A. Donker, H.G. van der Avoort, Continued seasonal circulation of enterovirus D68 in the Netherlands, 2011-2014, Euro Surveill. 19 (October (42)) (2014), pii: 20935

[6] K. Bragstad, K. Jakobsen, A.E. Rojahn, M.K. Skram, K. Vainio, M. HolbergPetersen, O. Hungnes, S.G. Dudman, A.B. Kran, High frequency of enterovirus D68 in children hospitalised with respiratory illness in Norway, autumn 2014 Influenza Other Respir. Viruses (December) (2014), http://dx.doi.org/10.1111/ irv.12300 [Epub ahead of print].

[7] A. Piralla, A. Girello, M. Grignani, M. Gozalo-Margüello, A. Marchi, G. Marseglia, F. Baldanti, Phylogenetic characterization of enterovirus 68 strains in patients with respiratory syndromes in Italy, J. Med. Virol. 86 (September (9)) (2014) 1590-1593.

[8] J. Garcia, V. Espejo, M. Nelson, M. Sovero, M.V. Villaran, J. Gomez, M. Barrantes, F. Sanchez, G. Comach, A.E. Arango, N. Aguayo, I.L. de Rivera, W. Chicaiza, M Jimenez, W. Aleman, F. Rodriguez, M.S. Gonzales, T.J. Kochel, E.S. Halsey, Human rhinoviruses and enteroviruses in influenza-like illness in Latin America, Virol. J. October (10) (2013) 305.

[9] Q.B. Lu, Y. Wo, H.Y. Wang, M.T. Wei, L. Zhang, H. Yang, E.M. Liu, T.Y. Li, Z.T. Zhao W. Liu, W.C. Cao, Detection of enterovirus 68 as one of the commonest types of enterovirus found in patients with acute respiratory tract infection in China, J Med. Microbiol. 63 (March (Pt 3)) (2014) 408-414

[10] A. Meijer, S. van der Sanden, B.E. Snijders, G. Jaramillo-Gutierrez, L. Bont, C.K. van der Ent, P. Overduin, S.L. Jenny, E. Jusic, H.G. van der Avoort, G.J. Smith, G.A Donker, M.P. Koopmans, Emergence and epidemic occurrence of enterovirus 68 respiratory infections in The Netherlands in 2010, Virology 423 (February (1)) (2012) 49-57.

[11] Centers for Disease Control and Prevention (CDC), Enterovirus surveillance United States, 2002-2004, MMWR Morb. Mortal. Wkly. Rep. 55 (February (6)) (2006) 153-156.

[12] A.L Greninger, S.N. Naccache, K. Messacar, A Clayton, G. Yu, S. Somasekar, S. Federman, D. Stryke, C. Anderson, S. Yagi, S. Messenger, D. Wadford, D. Xia, J.P. Watt, K. Van Haren, S.R. Dominguez, C. Glaser, G. Aldrovandi, C.Y. Chiu, A novel outbreak enterovirus D68 strain associated with acute flaccid myelitis cases in the USA (2012-14): a retrospective cohort study, Lancet Infect. Dis. (2015), http://dx.doi.org/10.1016/S1473-3099(15)70093-9, Published Online March 31.

[13] K. Messacar, T.L. Schreiner, J.A. Maloney, A. Wallace, J. Ludke, M.S. Oberste, W. A. Nix, C.C. Robinson, M.P. Glodé, M.J. Abzug, S.R. Dominguez, A cluster of acute flaccid paralysis and cranial nerve dysfunction temporally associated with an outbreak of enterovirus D68 in children in Colorado, USA, Lancet (January) (2015), pii: S0140-6736(14)62457-0 (Epub ahead of print)

[14] M.S. Oberste, K. Maher, D. Schnurr, M.R. Flemister, J.C. Lovchik, H. Peters, W. Ses sions, C. Kirk, N. Chatterjee, S. Fuller, J.M. Hanauer, M.A. Pallansch, Enterovirus 68 is associated with respiratory illness and shares biological features with both the enteroviruses and the rhinoviruses, J. Gen. Virol. 85 (September (Pt 9)) (2004) 2577-2584

[15] T. Smura, P. Ylipaasto, P. Klemola, S. Kaijalainen, L. Kyllönen, V. Sordi, L. Piemonti, M. Roivainen, Cellular tropism of human enterovirus D species serotypes EV-94, EV-70, and EV-68 in vitro: implications for pathogenesis, J. Med. Virol. 82 (November (11)) (2010) 1940-1949.

[16] S. Blomqvist, C. Savolainen, L. Råman, M. Roivainen, T. Hovi, Human rhinovirus 87 and enterovirus 68 represent a unique serotype with rhinovirus and enterovirus features, J. Clin. Microbiol. 40 (November (11)) (2002 4218-4223. 
[17] T. Imamura, A. Suzuki, S. Lupisan, T. Kamigaki, M. Okamoto, C.N. Roy, R. Olveda, H. Oshitani, Detection of enterovirus 68 in serum from pediatric patients with pneumonia and their clinical outcomes, Influenza Other Respir. Viruses 8 (January (1)) (2014) 21-24.

[18] J. Rahamat-Langendoen, A. Riezebos-Brilman, R. Borger, R. van der Heide, A. Brandenburg, E. Schölvinck, H.G. Niesters, Upsurge of human enterovirus 68 infections in patients with severe respiratory tract infections, J. Clin. Virol. 52 (October (2)) (2011) 103-106.

[19] F. Renois, A. Bouin, L. Andreoletti, Enterovirus 68 in pediatric patients hospitalized for acute airway diseases, J. Clin. Microbiol. 51 (February (2)) (2013) 640-643.

[20] T. Ikeda, K. Mizuta, C. Abiko, Y. Aoki, T. Itagaki, F. Katsushima, Y. Katsushima, Y. Matsuzaki, N. Fuji, T. Imamura, H. Oshitani, M. Noda, H. Kimura, T. Ahiko, Acute respiratory infections due to enterovirus 68 in Yamagata, Japan between 2005 and 2010, Microbiol. Immunol. 56 (February (2)) (2012) 139-143.

[21] T. Imamura, N. Fuji, A. Suzuki, R. Tamaki, M. Saito, R. Aniceto, H. Galang, L. Sombrero, S. Lupisan, H. Oshitani, Enterovirus 68 among children with severe acute respiratory infection, the Philippines, Emerg. Infect. Dis. 17 (August (8)) (2011) 1430-1435.

[22] G. Jaramillo-Gutierrez, K.S. Benschop, E.C. Claas, A.S. de Jong, A.M. van Loon, S.D. Pas, O. Pontesilli, J.W. Rossen, C.M. Swanink, S. Thijsen, A.G. van der Zanden, H.C. van der Avoort, M.P. Koopmans, A. Meijer, September through October 2010 multi-centre study in the Netherlands examining laboratory ability to detect enterovirus 68, an emerging respiratory pathogen, J. Virol. Methods 190 (June $(1-2))(2013) 53-62$.

[23] M.A. Pretorius, S. Tempia, F.K. Treurnicht, S. Walaza, A.L. Cohen, J. Moyes, O. Hellferscee, E. Variava, H. Dawood, M. Chhagan, S. Haffjee, S.A. Madhi, C. Cohen, M. Venter, Genetic diversity and molecular epidemiology of human rhinoviruses in South Africa, Influenza Other Respir. Viruses 8 (September (5)) (2014) 567-573

[24] T. Cutino-Moguel, I.L. Lauinger, S. Srivastava, M. Zuckerman, C.Y. Tong, S. Devereux, Analysis of a potential cluster of rhinovirus infections in patients and staff on two haemato-oncology wards, J. Clin. Virol. 60 (May (1)) (2014) 57-59.

[25] W.A. Nix, M.S. Oberste, M.A. Pallansch, Sensitive, seminested PCR amplification of VP1 sequences for direct identification of all enterovirus serotypes from original clinical specimens, J. Clin. Microbiol. 44 (August (8)) (2006) 26982704.

[26] R. Tokarz, C. Firth, S.A. Madhi, S.R. Howie, W. Wu, A.A. Sall, S. Haq, T. Briese, W.I. Lipkin, Worldwide emergence of multiple clades of enterovirus 68, J. Gen. Virol. 93 (September (Pt 9)) (2012) 1952-1958.

[27] T. Imamura, M. Okamoto, S. Nakakita, A. Suzuki, M. Saito, R. Tamaki, S. Lupisan, C.N. Roy, H. Hiramatsu, K.E. Sugawara, K. Mizuta, Y. Matsuzaki, Y. Suzuki, H. Oshitani, Antigenic and receptor binding properties of enterovirus 68, J. Virol. 88 (March (5)) (2014) 2374-2384
[28] S. Hasegawa, R. Hirano, R. Okamoto-Nakagawa, T. Ichiyama, K. Shirabe, Enterovirus 68 infection in children with asthma attacks: virus-induced asthma in Japanese children, Allergy 66 (December (12)) (2011) 1618-1620.

[29] A. Piralla, D. Lilleri, A Sarasini, A. Marchi, M. Zecca, M. Stronati, F. Baldanti, G. Gerna, Human rhinovirus and human respiratory enterovirus (EV68 and EV104) infections in hospitalized patients in Italy, 2008-2009, Diagn. Microbiol. Infect. Dis. 73 (June (2)) (2012) 162-167.

[30] J.S. Casalegno, M. Bouscambert-Duchamp, F. Morfin, B. Lina, V. Escuret, Rhinoviruses, A(H1N1)v, RVS: the race for hivernal pandemics, France 2009-2010, Euro Surveill. 14 (November (44)) (2009), pii: 19390.

[31] A. Waghmare, S.A. Pergam, K.R. Jerome, J.A. Englund, M. Boeckh, J. Kuypers, Clinical disease due to enterovirus D68 in adult hematologic malignancy patients and hematopoietic cell transplant recipients, Blood (January) (2015), pii: blood2014-12-616516 [Epub ahead of print].

[32] N. Falah, R. Montserret, V. Lelogeais, I. Schuffenecker, B. Lina, J.C. Cortay, S. Violot, Blocking human enterovirus 71 replication by targeting viral $2 \mathrm{~A}$ protease, J. Antimicrob. Chemother. 67 (December (12)) (2012) 2865-2869.

[33] N. Falah, S. Violot, D. Décimo, F. Berri, M.L. Foucault-Grunenwald, T. Ohlmann, I. Schuffenecker, F. Morfin, B. Lina, B. Riteau, J.C. Cortay, Ex vivo and in vivo inhibition of human rhinovirus replication by a new pseudosubstrate of viral 2A protease, J. Virol. 86 (January (2)) (2012) 691-704.

[34] Y. Liu, J. Sheng, A. Fokine, G. Meng, W.H. Shin, F. Long, R.J. Kuhn, D. Kihara, M.G. Rossmann, Virus structure. Structure and inhibition of EV-D68, a virus that causes respiratory illness in children, Science 347 (January (6217)) (2015) 71-74.

[35] A. Bal, I. Schuffenecker, J.S. Casalegno, L. Josset, M. Valette, N. Armand, P. Bellia Dhondt, V. Escuret, B. Lina, Enterovirus D68 nosocomial outbreak in elderly people, France, 2014, Clin. Microbiol. Infect. 21 (August (8)) (2015) e61-e62.

[36] M. Lang, A. Mirand, N. Savy, C. Henquell, S. Maridet, R. Perignon, A. Labbe, H. Peigue-Lafeuille, Acute flaccid paralysis following enterovirus D68 associated pneumonia, France, 2014, Euro Surveill. 19 (November (44)) (2014), pii: 20952.

[37] A. Mirand, H. Peigue-Lafeuille, Acute flaccid myelitis and enteroviruses: an ongoing story, Lancet (January) (2015), http://dx.doi.org/10.1016/S01406736(15)60121-0, pii: S0140-6736(15)60121-0 [Epub ahead of print].

[38] S.M. Opanda, F. Wamunyokoli, S. Khamadi, R. Coldren, W.D. Bulimo, Genetic diversity of human enterovirus 68 strains isolated in Kenya using the hypervariable 3'-end of VP1 gene, PLOS ONE 9 (July (7)) (2014) e102866.

[39] T. Imamura, A. Suzuki, S. Lupisan, M. Okamoto, R. Aniceto, R.J. Egos, E.E. Daya, R. Tamaki, M. Saito, N. Fuji, C.N. Roy, J.M. Opinion, A.V. Santo, N.G. Macalalad, A. Tandoc 3rd, L. Sombrero, R. Olveda, H. Oshitani, Molecular evolution of enterovirus 68 detected in the Philippines, PLOS ONE 8 (September (9)) (2013) e74221.

[40] S. Esposito, A. Zampiero, L. Ruggiero, B. Madini, H. Niesters, N. Principi, Enterovirus D68-associated community-acquired pneumonia in children living in Milan, Italy, J. Clin. Virol. (May) (2015) 94-96. 